\title{
The antitumor effect of PLK1 and HSF1 double knockdown on human oral carcinoma cells
}

\author{
SOO-A KIM ${ }^{1}$, SEONG-MIN KWON ${ }^{2}$, JUNG-HOON YOON ${ }^{2}$ and SANG-GUN AHN ${ }^{2}$ \\ ${ }^{1}$ Department of Biochemistry, Dongguk University College of Oriental Medicine, Gyeongju 780-714; \\ ${ }^{2}$ Department of Pathology, Chosun University College of Dentistry, Gwangju 501-759, Korea
}

Received September 1, 2009; Accepted December 22, 2009

DOI: 10.3892/ijo_00000564

\begin{abstract}
High levels of mitotic progression-associated PLK1 and stress-associated HSF1 have been observed in various human cancers. In the present study, we investigated the effects of PLK1 and HSF1 knockdown on the proliferation of oral cancer cells using small interfering RNA. In human oral squamous cell carcinoma (SCC) tissues, the levels of PLK1 and HSF1 were higher compared to normal tissues. The expression levels of PLK1 and HSF1 were also elevated in the human oral SCC cell lines FaDu and HEp-2. Disruption of PLK1 induced cell cycle arrest at G2/M phase as well as apoptosis in oral cancer cells. Interestingly, knockdown of both PLK1 and HSF1 expression decreased cell proliferation while increasing apoptotic cell death in synergistic fashion. These results establish the potential value of PLK1 and HSF1 as targets for oral cancer therapy.
\end{abstract}

\section{Introduction}

Polo-like kinase 1 (PLK1) is a serine/threonine protein kinase that acts as an important cell signaling regulator. It is involved in the regulation of the cell cycle, centrosome maturation, regulation of anaphase-promotion complex and bipolar spindle formation (1-3). Recent findings suggest that the expression of PLK1 is elevated in many types of human cancer, including head and neck squamous cell carcinoma, prostate cancer, pancreatic cancer, breast cancer, colorectal cancer and ovarian cancer (4-6). It has been suggested that PLK1 plays a key role in $\mathrm{G} 2 / \mathrm{M}$ phase progression by regulating the targeting of cyclin B and the activity of cdc25C phosphatase $(7,8)$. PLK1 also binds to the tumor suppressor p53 in mammalian cells, inhibiting its transactivation as well as pro-apoptotic function (9). Several studies have recently shown that inhibition of

Correspondence to: Dr Jung-Hoon Yoon or Dr Sang-Gun Ahn, Department of Pathology, Chosun University College of Dentistry, 375 Seosuk-dong, Dong-gu, Gwangju 501-759, Korea

E-mail: jhyoon@chosun.ac.kr

E-mail: ahnsg@chosun.ac.kr

Key words: Polo-like kinase 1, Heat shock factor 1, siRNA, oral cancer, apoptosis
PLK1 leads to cell cycle arrest, induction of apoptosis and suppression of cancer cell growth both in vitro and in vivo (10-13).

Heat shock factor 1 (HSF1) is a transcription factor that is strongly conserved from yeast to humans. HSF1 is responsible for the expression of a large class of heat shock proteins (Hsps), which serve to protect cells from damage as a result of cellular insults such as heat and oxidative stress $(14,15)$. Upon sensing stress, HSF1 undergoes a transition from a monomeric to a homotrimeric form, where it localizes to the nucleus, binds DNA and acts as a transactivator (16). Recent studies have reported that expression of HSF1 is observed in several human cancers such as prostate and colorectal cancer $(17,18)$. Additionally, the expression of Hsps becomes dysregulated in cancer tissues and cell lines. The aberrant expression of Hsp27, Hsp70 and Hsp90 reported in human malignant cancers of various origin correlates with the development of cancer, invasiveness, metastasis, resistance to chemotherapy, and radiation therapy (19-22). Therefore, it is strongly proposed that HSF1 and Hsps could be considered targets for novel cancer therapies.

Oral cancer is one of the fastest growing malignancies and particularly dangerous because of a high risk of producing secondary tumors. There are several types of oral cancers of which $90 \%$ are squamous cell carcinomas $(23,24)$. Despite enormous efforts for improvement, survival rates have remained unchanged over 20 years due to a lack of markers for early prognosis and the failure of advanced lesions to respond to chemotherapy.

In this study, we evaluated the relationship of PLK1 and HSF1 as targets for oral cancer by using siRNA-mediated gene silencing techniques. We demonstrate that knockdown of both PLK1 and HSF1 in oral squamous cell carcinoma cells strongly inhibits cell proliferation while inducing cell death. These findings establish the double knockdown of PLK1 and HSF1 as a potential strategy for cancer therapy.

\section{Materials and methods}

Cell culture. Human FaDu (hypopharyngeal cancer) and HEp-2 (laryngeal cancer) cells were obtained from the American Type Culture Collection (Manassas, VA). Cells were maintained in MEM medium supplemented with 5\% fetal bovine serum, $100 \mathrm{U} / \mathrm{ml}$ penicillin and $100 \mu \mathrm{g} / \mathrm{ml}$ streptomycin (Invitrogen, CA). Human immortalized normal 
oral keratinocyte (INOK) cells were supplied by Dr E.C. Kim (Wonkwang University, Iksan, Korea) and were maintained in $\mathrm{KGM}$ medium (Invitrogen). Cells were cultured at $37^{\circ} \mathrm{C}$ in a humidified atmosphere with $5 \% \mathrm{CO}_{2}$.

MTT assay. Briefly, cells were seeded on 12-well plates at a density of $1 \times 10^{5}$ cells $/ \mathrm{ml}$. Cells were then cultured overnight and transfected with siRNA for 24 or $48 \mathrm{~h}$. Cell proliferation was evaluated by 3-(4,5-dimethylthiazol-2-yl)-2,5-diphenyltetrazolium bromide (MTT) assay as previously described (25).

Immunohistochemistry. Tumors were acquired during the surgical procedure. Excised human oral squamous cell carcinomas were fixed in $10 \%$ buffered formalin and embedded in paraffin. Immunohistochemical staining was performed following the avidin-biotin complex method. Briefly, slides were deparaffinized and rehydrated. Endogenous peroxidase was blocked with $3 \%$ hydrogen peroxide $\left(\mathrm{H}_{2} \mathrm{O}_{2}\right)$ in methanol, and the sections were incubated with anti-PLK1 antibodies (Santa Cruz Biotechnology, Santa Cruz, CA) or anti-HSF1 antibodies (Santa Cruz Biotechnology). Immune reactions were visualized with 3,3'-diaminobenzidine and counterstained with Mayer's hematoxylin.

siRNA experiments. A 19-nucleotide double-stranded siRNA for HSF1 was generated using oligonucleotide (5'-TTCCTG ACCAAGCTGTGGA-3') and inserted into the 5'-XhoI and 3'-XbaI sites of the pSuppressorNeo vector (Imgenex, San Diego, CA). Cells were transfected with RNAi expression vector for HSF1 (pSuppressorNeo-HSF1) using FuGENE 6 reagent (Roche Molecular Biochemicals, Indianapolis, IN) according to the manufacturer's instructions. siRNA construct for PLK1 was obtained in the form of Silencer ${ }^{\circledR}$ select validated siRNA (Applied Biosystems, Foster City, CA). Cells were transfected with siRNA using X-tremeGENE siRNA Transfection Reagent (Roche Molecular Biochemicals) according to the manufacturer's instructions. Cells were harvested $24 \mathrm{~h}$ after transfection. Total cell lysates were separated by SDS-PAGE and analyzed by Western blot analysis as described below.

Western blot analysis. Total proteins $(50 \mu \mathrm{g})$ were resolved by SDS-PAGE and transferred onto PVDF membrane. After blocking, membranes were blotted with antibodies against PLK1, HSF1, Hsp70, Hsp90 or actin (Santa Cruz Biotechnology).

Assessment of cell cycle distribution and apoptosis. The effect of siRNA on cell cycle distribution was determined by flow cytometric analysis after staining cells with propidium iodide. Briefly, $1 \times 10^{6}$ cells were seeded on a $60 \mathrm{~mm}$ dish and allowed to attach overnight. Cells were transfected with siRNA constructs for $24 \mathrm{~h}$. After washing with cold PBS, cells were fixed with $100 \%$ ethanol at $-20^{\circ} \mathrm{C}$. The cells were then treated with one unit of DNase-free RNase and incubated for $30 \mathrm{~min}$ at $37^{\circ} \mathrm{C}$. Propidium iodide was added directly to the cell suspension and then analyzed using a Cell Lab Quanta ${ }^{\mathrm{TM}}$ SC flow cytometer (Beckman Coulter Inc., Fullerton, CA). Apoptotic cell death was identified by labeling the cells with Annexin V-FITC and propidium iodide using the Vybrant ${ }^{\circledR}$

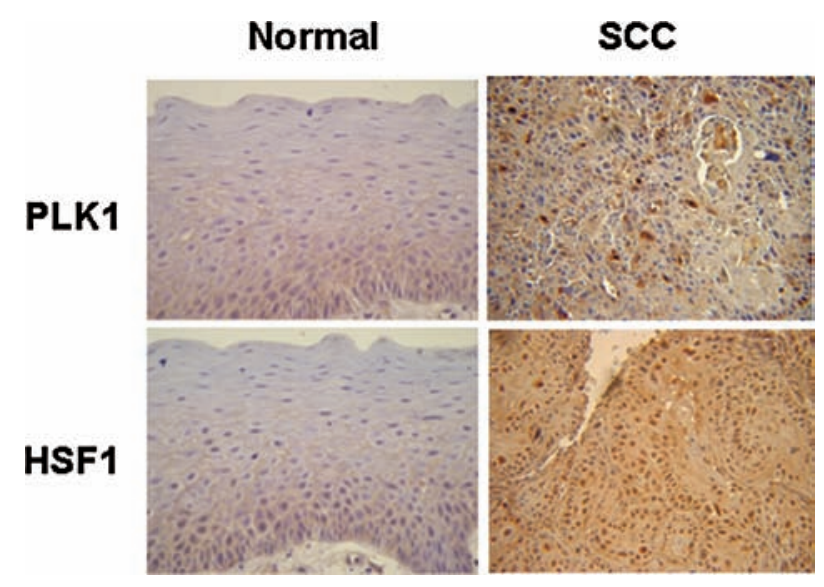

Figure 1. Immunohistochemical staining for PLK1 and HSF1 in paraffinembedded human SCC tissues.

A

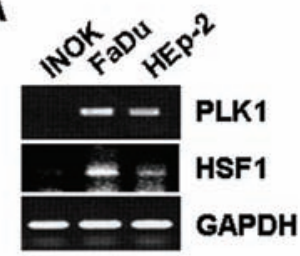

B

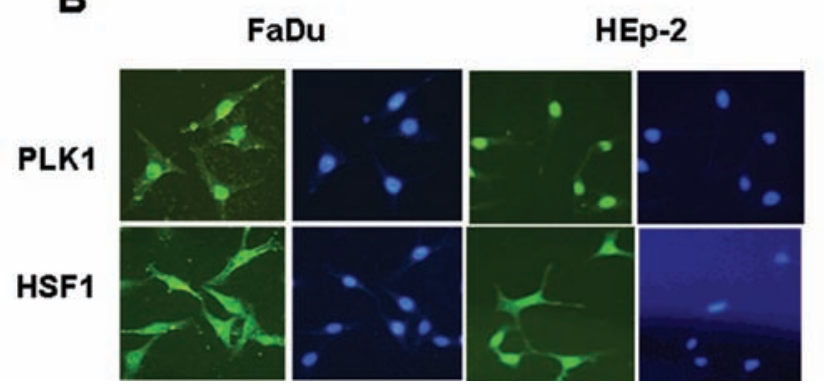

Figure 2. The expression and localization of PLK1 and HSF1 in human SCC cells. (A) Total RNAs prepared from INOK, FaDu and HEp-2 cells were subjected to RT-PCR analysis using primers specific for PLK1 and HSF1. GAPDH was used as an internal control. (B) FaDu and HEp-2 cells were transiently transfected with pCDNA3.1-FLAG-PLK1 or pCDNA3.1-FLAGHSF1 mammalian expression vectors. At $24 \mathrm{~h}$ after transfection, cells were fixed and immunostained using anti-FLAG antibodies. Nuclei were stained with DAPI (4,6-diamidino-2-phenylindole). (C) PLK1 kinase activity was measured in cytoplasmic (C) or nuclear (N) fractions using a CycLex Polo-like kinase 1 assay kit. The absorbance was measured at a wavelength of $450 \mathrm{~nm}$ and data were expressed as the means \pm SD of the results from three separate experiments.

Apoptosis Assay Kit (Invitrogen) according to the manufacturer's instruction.

Kinase assay. The kinase activity of PLK1 and Cdc2/cyclin B complex was measured using $\mathrm{CycLex}^{\circledR}$ PLK1 and CycLex Cdc2/cyclin B kinase assay kits (CycLex Co., Ltd., Nagano, Japan) according to the manufacturer's instructions. The absorbance was measured at a wavelength of $450 \mathrm{~nm}$. The results represent the amount of phosphorylated substrate. 

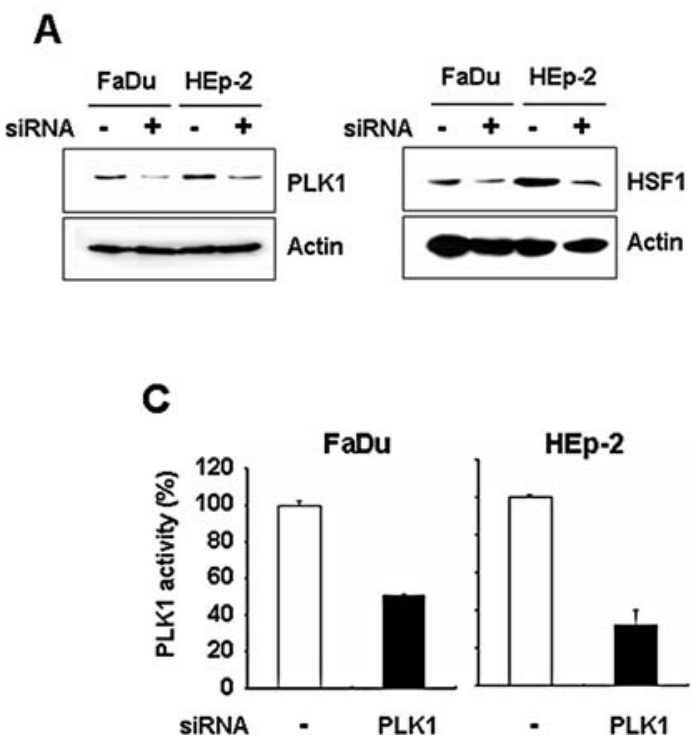

B

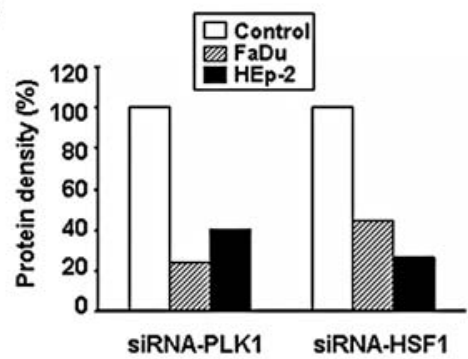

Figure 3. Depletion of endogenous PLK1 or HSF1 by siRNA. (A) Cells were transfected with siRNA for PLK1 or RNAi expression vector for HSF1 (pSuppressorNeo-HSF1). At $24 \mathrm{~h}$ after transfection, total cell extracts were prepared. Protein levels of PLK1 and HSF1 were determined by Western blot analysis. (B) Quantitative analyses of PLK1 and HSF1 protein levels were obtained by densitometric scanning of Western blots. Protein levels were expressed as $\%$ of control after normalization with actin. (C) At $24 \mathrm{~h}$ after transfection with siRNA for PLK1, total cell extracts were prepared and the PLK1 kinase activity was measured at $450 \mathrm{~nm}$ using a CycLex Polo-like kinase 1 assay kit. The data were expressed as the means \pm SD of the results from three separate experiments.

\section{Results}

PLK1 and HSF1 are overexpressed in oral SCC tissues and oral cancer cells. To investigate the expression levels of PLK1 and HSF1 in human oral squamous cell carcinoma (SCC), we performed an immunohistochemistry assay for PLK1 and HSF1 in five oral SCC tissues. As shown in Fig. 1, the level of PLK1 was significantly higher in oral SCC compared with that of normal tissue. This result is consistent with high levels of PLK1 previously observed in head and neck squamous cell cancers (HNSCCs) (5). We observed the expression level of HSF1 in oral SCC tissues for the first time and then demonstrated the overexpression of HSF1 in oral SCC (Fig. 1).

The expression levels of PLK1 and HSF1 were also assessed in the human oral SCC cell lines FaDu and HEp-2. As shown in Fig. 2A, the expression levels of PLK1 and HSF1 were much higher in both types of oral cancer cells than in immortalized normal oral keratinocyte (INOK) cells. To evaluate the subcellular localization of PLK1 and HSF1, cells were transfected with pCDNA3.1-FLAG-PLK1 or pCDNA3.1-FLAG-HSF1 mammalian expression vectors. While HSF1 was localized in the cytoplasm and nucleus of FaDu and HEp-2 cells, PLK1 was primarily localized in the nucleus (Fig. 2B). We next examined PLK1 activity in the cytoplasmic and nuclear fractions. As shown in Fig. 2C, PLK1 activity was higher in the nucleus compared to the cytoplasm, suggesting PLK1 may play a major role in the nucleus.

Depletion of PLK1 and HSF1 inhibits cell proliferation and decreases cell viability. To specifically deplete the expression of PLK1 and HSF1 in oral cancer cells, we employed siRNA techniques. Transfection of RNAi expression vectors for
A
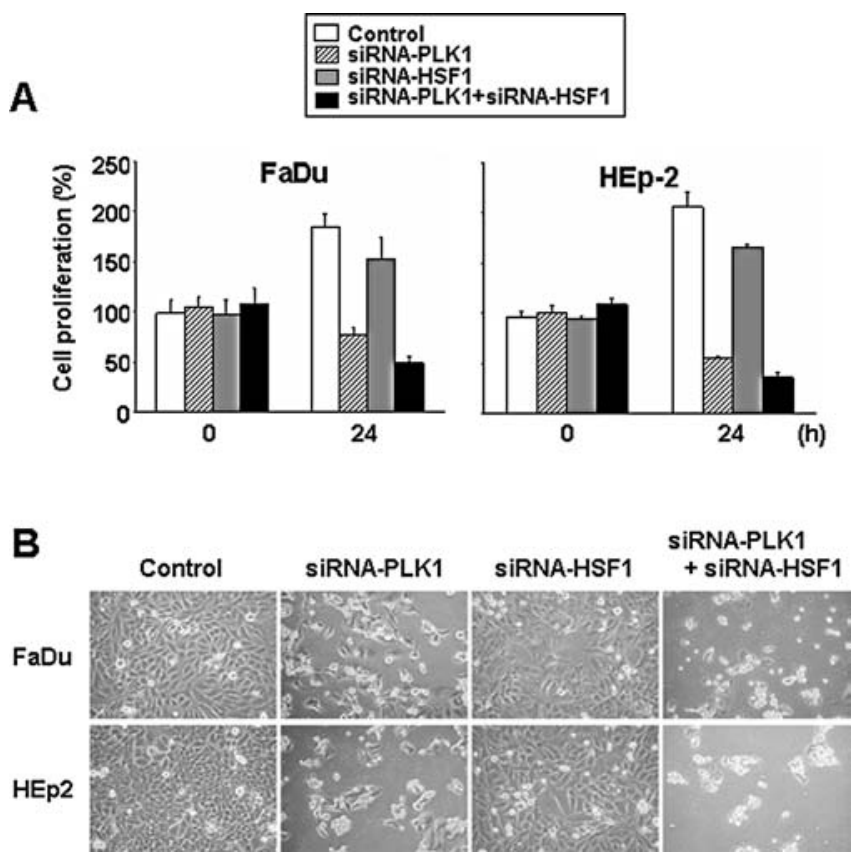

Figure 4. Effects of PLK1 and/or HSF1 depletion on the proliferation of FaDu and HEp-2 cells. Cells were transfected with siRNA for PLK1 and/or RNAi expression vector for HSF1 (pSuppressorNeo-HSF1). At $24 \mathrm{~h}$ after transfection, cell proliferation was measured by MTT assay (A) and cells were examined by inverted microscopy (B).

HSF1 (pSppressorNeo-HSF1) or PLK1 oligonucleotides led to the inhibition of endogenous HSF1 or PLK1, respectively in FaDu and HEp-2 cells (Fig. 3A and B). The activity of PLK1 was efficiently inhibited by 50 and $70 \%$ in siRNAtransfected $\mathrm{FaDu}$ and HEp-2 cells, respectively (Fig. 3C).

We next determined whether PLK1 and/or HSF1 depletion influences cell proliferation. As shown in Fig. 4, depletion of 
A

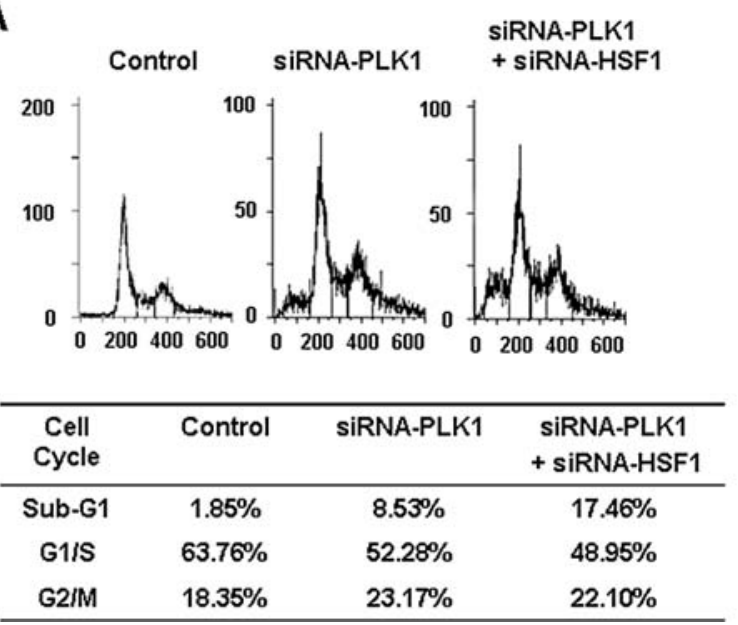

B

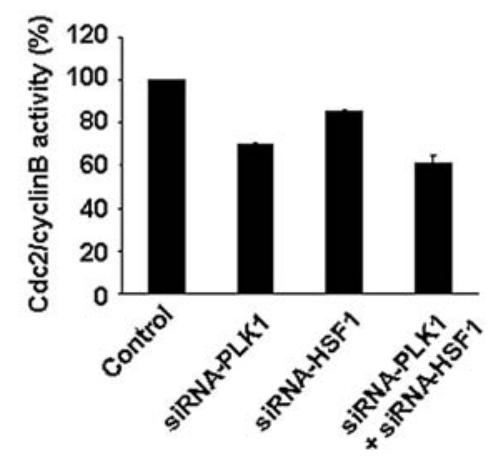

Figure 5. PLK1 depletion induces cell cycle arrest at G2/M phase. (A) Cells were transfected with siRNA for PLK1 and/or pSuppressorNeo-HSF1 for 12 h. Cell cycle distribution was monitored by flow cytometry. (B) Cells were transfected with siRNA for PLK1 and/or pSuppressorNeo-HSF1 for 24 h. Total cell lysates were prepared and the Cdc2/cyclin B complex activity was measured using a CycLex Cdc2/cyclin B kinase assay kit. The data are expressed as the means $\pm \mathrm{SD}$ of results from three separate experiments.

A
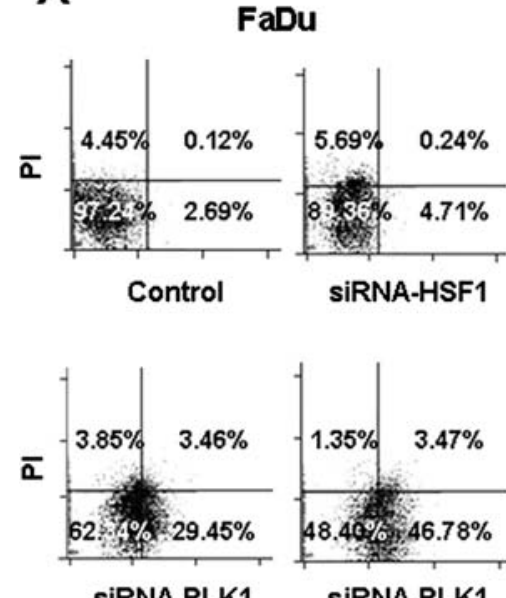

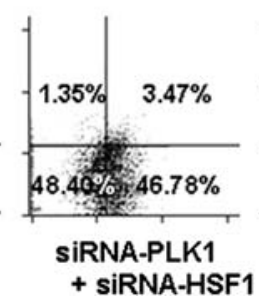

B
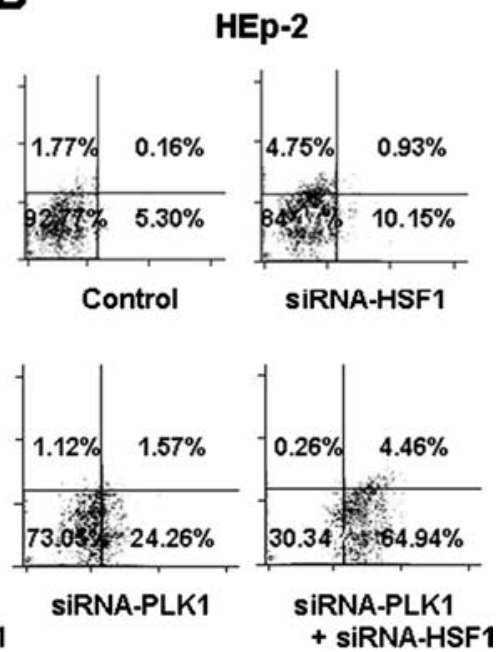

Figure 6. PLK1 and HSF1 double depletion synergistically induces apoptosis. Cells were transfected with siRNA for PLK1 and/or pSuppressorNeo-HSF1 for $24 \mathrm{~h}$. Apoptosis was assessed by flow cytometry by staining the cells with Annexin-V-FLUOS and propidium iodide.

HSF1 showed slightly decreased cell proliferation. However, depletion of PLK1 largely decreased the cell proliferation and dramatically changed the cell phenotype. Interestingly, depletion of both PLK1 and HSF1 resulted in a synergistic decrease of cell proliferation and showed the increased phenotype of cell death compared with PLK1-depleted cells (Fig. 4).

PLK1 depletion induces G2/M phase cell cycle arrest. Previous studies have shown that PLK1 is an important regulator for mitotic entry. To evaluate the effect of PLK1 and HSF1 double knockdown on cell cycle progression, cells were transfected with siRNA for PLK1 and/or HSF1 for $12 \mathrm{~h}$ and flow cytometric analysis was performed. As shown in Fig. 5A, depletion of PLK1 increased the number of cells arrested in $\mathrm{G} 2 / \mathrm{M}$ phase to 23.17 from $18.35 \%$ in control cells. PLK1 acts as a cell cycle regulator to directly target many key cell cycle regulators, such as $\mathrm{Cdc} 2 /$ cyclin $\mathrm{B}$ complex. We found that the activity of Cdc2/cyclin B complex in PLK1-depleted cells was $30.2 \%$ lower than that of control cells (Fig. 5B). Cells depleted of PLK1 and HSF1 did not show any difference in Cdc2/cyclin B activity compared with PLK1-depleted cells (Fig. 5).

PLK1 and HSF1 double knockdown synergistically induces apoptosis. More importantly, cells accumulated in sub-G1 phase (apoptotic cells) were increased from $1.85 \%$ in control cells to $8.53 \%$ in PLK1-depleted cells (Fig. 5A). When cells were subjected to double knockdown of PLK1 and HSF1, sub-G1 phase cells were increased markedly up to $17.46 \%$ (Fig. 5A). These data demonstrate that depletion of both PLK1 and HSF1 significantly increases cellular sensitivity to apoptosis. We further analyzed the effect of PLK1 and HSF1 double knockdown on cell death using flow cytometry. As shown in Fig. 6, PLK1 depletion resulted in an increase in apoptotic cell death from 2.69 to $29.45 \%$ in $\mathrm{FaDu}$ cells. 


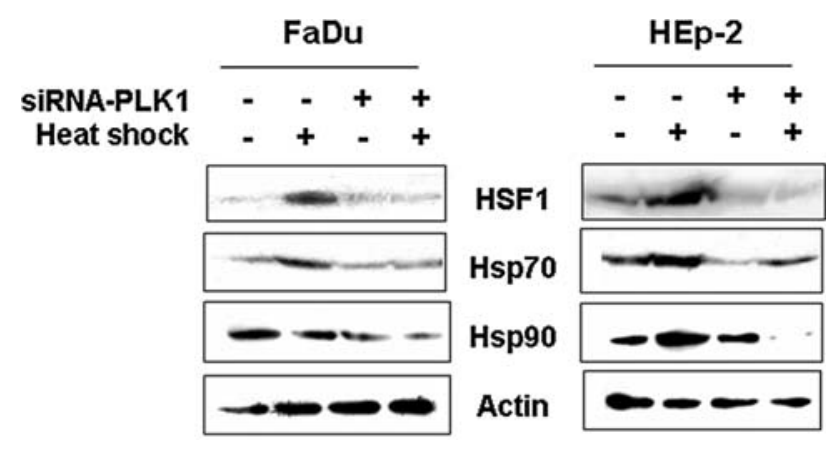

Figure 7. Effects of PLK1 depletion on heat shock response. Cells were transfected with siRNA constructs for PLK1. At $24 \mathrm{~h}$ after transfection, cells were treated with or without heat shock at $42^{\circ} \mathrm{C}$ for $1 \mathrm{~h}$ and recovered at $37^{\circ} \mathrm{C}$ for $6 \mathrm{~h}$. Total cell lysates were prepared and the expression levels of HSF1, Hsp70, Hsp90 and actin were detected by Western blot analysis.

HEp-2 cells depleted of PLK1 produced a similar result with apoptotic cell death being increased from 5.30 in control cells to $24.26 \%$. On the contrary, depletion of HSF1 led to a slight increase in apoptotic cell death (Fig. 6). However, when the cells were cotransfected with siRNA for PLK1 and HSF1, apoptotic cell death was increased markedly up to 46.78 and $64.94 \%$ in $\mathrm{FaDu}$ and HEp-2 cells, respectively. These results suggest that the depletion of both PLK1 and HSF1 synergistically inhibits cell proliferation while inducing apoptosis in oral carcinoma cells.

PLK1 depletion inhibits the induction of HSF1 and Hsps by heat stress. Recently, we have shown that PLK1 phosphorylates HSF1 and regulates its nuclear translocation (26). Therefore, we next investigated whether the stress-induced activation of HSF1 was affected by PLK1 depletion. The cells were transfected with siRNA specific for PLK1, heat shocked at $42^{\circ} \mathrm{C}$ for $1 \mathrm{~h}$ and then allowed to recover at $37^{\circ} \mathrm{C}$ for $6 \mathrm{~h}$. As expected, heat shock treatment induced the expression of HSF1, Hsp70 and Hsp90 in untransfected control cells (Fig. 7). However, the depletion of PLK1 significantly inhibited the induction of heat shock responsive proteins, suggesting that PLK1 is a key regulator for HSF1 activation (Fig. 7).

\section{Discussion}

PLK1 plays an important role in cellular mitotic events (1-3), and its overexpression is closely related with tumor proliferation (4-6). HSF1 plays a broader role in cellular signaling beyond its well-known roles in stress response and cancer development $(14,27)$. Overexpression of HSF1 enhances the survival capacity and chemoresistance of cancer cells. In this study, we demonstrated high levels of PLK1 and HSF1 in oral SCC as well as in oral cancer cells. Furthermore, suppression of PLK1 and HSF1 expression produced antitumor effects in oral cancer cells such as loss of cell viability, cell cycle arrest and apoptosis.

We showed that PLK1 is predominantly localized in the nucleus and that its activity is higher in nucleus than in cytoplasm (Fig. 2). These results suggest that PLK1 may regulate nuclear proteins and/or transcription factors in the nucleus. Previous studies have shown that PLK1 phospho- rylates various proteins such as cyclin B1 and Cdc25C. PLK1 also promotes the nuclear entry of the $\mathrm{Cdc} 2 /$ cyclin $\mathrm{B}$ complex, followed by its direct activation during $\mathrm{G} 2 / \mathrm{M}$ phase by phosphorylated Cdc25C $(7,8)$. We showed that the activity of the Cdc2/cyclin B complex was 30.2\% lower in PLK1-depleted FaDu cells than in control cells (Fig. 5B). In contrast to our results, Liu and Erikson suggested that PLK1 depletion increases Cdc2/cyclin B complex activity in HeLa cells (28). However, Fink et al showed that inhibition of PLK1 activity exerts contrary effects depending on cell type (29). Current studies in our laboratory are underway to explore the signaling pathway modulated by PLK1 in oral cancer cells.

The aberrant expression of HSF1 and Hsps has been observed in aggressively malignant human cancer tissues (15-19). It is also reported that PLK1 is associated with Hsp90 and this relationship is important to centrosome function, cell division and may play a role in certain oncogenic events $(30,31)$. Previously, we demonstrated that PLK1 phosphorylates and thereby regulates the nuclear translocation of HSF1 (26). Therefore, we assessed whether HSF1 activation is affected by PLK1 depletion. The results showed that depletion of PLK1 significantly inhibits the expression of HSF1 as well as Hsp70 and Hsp90, the major targets of HSF1 (Fig. 7). This result strongly suggests that Hsp induction and PLK1 activation is closely related in oral cancer cells. We clearly showed that the combination of selective silencing of PLK1 and HSF1 synergistically inhibits cell proliferation and induces cell death in PLK1 and HSF1 double knockdown cells.

In conclusion, the results of this study, using siRNAmediated gene silencing of PLK1 and HSF1, showed strong inhibition of cell proliferation, cell cycle arrest and induction of apoptosis in oral cancer cells, thereby demonstrating its potential value as a target for oral cancer therapy.

\section{Acknowledgements}

S.G. Ahn was supported by the National R\&D Program for Cancer Control, Ministry of Health \& Welfare, Republic of Korea (00000355) and the Korea Science and Engineering Foundation (KOSEF) grant funded by the Korea government (MOST) (no. R13-2008-010-01001-0). S.A. Kim was supported by the Korea Research Foundation Grant funded by the Korean Government (MOEHRD) (KRF-2007-531E00009).

\section{References}

1. Donaldson MM, Tavares AA, Hagan IM, Nigg EA and Glover DM: The mitotic roles of Polo-like kinase. J Cell Sci 114: 2357-2358, 2001.

2. Nigg EA: Polo-like kinases: positive regulators of cell division from start to finish. Curr Opin Cell Biol 10: 776-783, 1998.

3. Mayor T, Meraldi P, Stierhof YD, Nigg EA and Fry AM: Protein kinases in control of the centrosome cycle. FEBS Lett 452: 92-95, 1999.

4. Noriyuki T, Ryoji H, Jun Y and Isao M: Polo-like kinases (Plks) and cancer. Oncogene 24: 287-291, 2005.

5. Knecht R, Elez R, Oechler M, Solbach C, VonIlberg C and Strebhardt K: Prognostic significance of polo-like kinase (PLK) expression in squamous cell carcinomas of the head and neck. Cancer Res 59: 2794-2797, 1999.

6. Takahashi T, Sano B, Nagata T, Kato H, Sugiyama Y, Kunieda K, Kimura M, Okano Y and Saji S: Polo-like kinase 1 (PLK1) is overexpressed in primary colorectal cancers. Cancer Sci 94: 148-152, 2003. 
7. Toyoshima-Morimoto F, Taniguchi E, Shinya N, Iwamatsu A and Nishida E: Polo-like kinase 1 phosphorylates cyclin B1 and targets it to the nucleus during prophase. Nature 410: 215-220, 2001.

8. Roshak AK, Capper EA, Imburgia C, Fornwald J, Scott G and Marshall LA: The human polo-like kinase, PLK, regulates cdc2/cyclin B through phosphorylation and activation of the cdc25C phosphatase. Cell Signal 12: 405-411, 2000.

9. Ando K, Ozaki T, Yamamoto H, Furuya K, Hosoda M, Hayashi S, Fukuzawa M and Nakagawara A: Polo-like kinase 1 (Plk1) inhibits p53 function by physical interaction and phosphorylation. J Biol Chem 279: 25549-25561, 2004.

10. Guan R, Tapang P, Leverson JD, Albert D, Giranda VL and Luo Y: Small interfering RNA-mediated Polo-like kinase 1 depletion preferentially reduces the survival of p53-defective, oncogenic transformed cells and inhibits tumor growth in animals. Cancer Res 64: 2698-2704, 2005.

11. Nogawa M, Yuasa T, Kimura S, Tanaka M, Kuroda J, Sato K, Yokota A, Segawa H, Toda Y, Kageyama S, Yoshiki T, Okada $\mathrm{Y}$ and Maekawa T: Intravesical administration of small interfering RNA targeting PLK-1 successfully prevents the growth of bladder cancer. J Clin Invest 115: 978-985, 2005.

12. Spankuch-Schmitt B, Bereiter-Hahn J, Kaufmann M and Strebhardt K: Effect of RNA silencing of polo-like kinase-1 (PLK1) on apoptosis and spindle formation in human cancer cells. J Natl Cancer Inst 94: 1863-1877, 2002.

13. Liu X and Erikson RL: Polo-like kinase (Plk) 1 depletion induces apoptosis in cancer cells. Proc Natl Acad Sci USA 100: 5789-5794, 2003.

14. Sorger PK: Heat shock factor and the heat shock response. Cell 65: 363-366, 1991.

15. Morano KA and Thiele DJ: Heat shock factor function and regulation in response to cellular stress, growth and differentiation signals. Gene Exp 7: 271-282, 1999.

16. Morimoto RI, Jurivich DA, Kroeger PE, Mathur SK, Murphy SP, Nakai A, Sarge K, Abravaya K and Sistonen LT: Regulation of heat shock gene transcription by a family of heat shock factors. In: The Biology of Heat Shock Proteins and Molecular Chaperones. Morimoto RI, Tisieres A and Georgopoulos C (eds.) Cold Spring Harbor Press, New York, pp417-455, 1994.

17. Hoang AT, Huang J, Rudra-Ganguly N, Zheng J, Powell WC, Rabindran SK, Wu C and Roy-Burman P: A novel association between the human heat shock transcription factor 1 (HSF1) and prostate adenocarcinoma. Am J Pathol 156: 857-864, 2000.

18. Cen H, Zheng S, Fang YM, Tang XP and Dong Q: Induction of HSF1 expression is associated with sporadic colorectal cancer. World J Gastroenterol 10: 3122-3126, 2004.
19. Tang D, Khaleque MA, Jones EL, Theriault JR, Li C, Wong WH, Stevenson MA and Calderwood SK: Expression of heat shock proteins and heat shock protein messenger ribonucleic acid in human prostate carcinoma in vitro and in tumors in vivo. Cell Stress Chaperones 10: 46-58, 2005.

20. Ciocca DR and Vargas-Roig LM: Hsp27 as a prognostic and predictive factor in cancer. Prog Mol Subcell Biol 28: 205-218, 2002.

21. Ricaniadis N, Kataki A, Agnantis N, Androulakis G and Karakousis CP: Long-term prognostic significance of HSP-70, c-myc and HLS-DR expression in patients with malignant melanoma. Eur J Surg Oncol 27: 88-93, 2001.

22. Strik HM, Weller M, Frank B, Hermisson M, Deininger MH, Dichgans J and Meyermann R: Heat shock protein expression in human gliomas. Anticancer Res 20: 4457-4462, 2000.

23. Canto MT and Devesa SS: Oral cavity and pharynx cancer incidence rates in the United States 1975-1998. Oral Oncol 38: 610-617, 2002.

24. Mignogna MD, Fedele S and Russo LL: The World Cancer Report and the burden of oral cancer. Eur J Cancer Prev 13: 139-142, 2004.

25. Kim SA, Kim YC, Kim SW, Lee SH, Min JJ, Ahn SG and Yoon JH: Antitumor activity of novel indirubin derivatives in rat tumor model. Clin Cancer Res 13: 253-259, 2007.

26. Kim SA, Yoon JH, Lee SH and Ahn SG: Polo-like kinase 1 phosphorylates heat shock transcription factor 1 and mediates its nuclear translocation during heat stress. J Biol Chem 280: 12653-12657, 2005.

27. Rossi A, Ciafre S, Balsamo M, Pierimarchi P and Santoro MG: Targeting the heat shock factor 1 by RNA interference: a potent tool to enhance hyperthermochemotherapy efficacy in cervical cancer. Cancer Res 66: 7678-7685, 2006.

28. Liu X and Erikson RL: Activation of Cdc2/cyclin B and inhibition of centrosome amplification in cells depleted of Plk1 by siRNA. Proc Natl Acad Sci USA 99: 8672-8676, 2002.

29. Fink J, Sanders K, Rippl A, Finkernagel S, Beckers TL and Schmidt M: Cell type-dependent effects of Polo-like kinase 1 inhibition compared with targeted polo box interference in cancer cell lines. Mol Cancer Ther 6: 3189-3197, 2007.

30. De Carcer G, Do Carmo Avides M, Lallena MJ, Glover DM and Gonzalez C: Requirement of Hsp90 for centrosomal function reflects its regulation of Polo kinase stability. EMBO J 20: 2878-2884, 2001

31. Simizu S and Osada H: Mutations in the Plk gene lead to instability of Plk protein in human tumour cell lines. Nat Cell Biol 2: 852-854, 2000. 